\title{
The Development of Advanced-Technology Automated/Robotic Telescope Systems and the Future of Small-Telescope Astronomy
}

\author{
Richard J. Williams \& James Mulherin \\ Torus Technologies, 3007 Sierra Court, Iowa City, IA 52244, USA
}

\begin{abstract}
During the 1990s groups at universities around the world developed small working automated/robotic telescopes that proved the feasibility of using such systems for education and research projects. A few of the more successful projects such as the Bradford Robotic Observatory in the United Kingdom and the University of Iowa's Automated Telescope Facility (AFT) and Iowa Robotic Observatory (IRO) programs proved how useful and powerful these systems can be in practice. This paper describes how one company, Torus Technologies, developed hardware and software technologies to create the most advanced integrated small automated/robotic telescope systems in the world. These systems were designed from the "bottom up" to be automated/robotic telescopes capable of operating an entire observatory including domes, CCD cameras, and other peripheral equipment.

Automated/robotic telescopes can play a major role in enabling small colleges and universities, especially in developing countries, to actively participate in serious "hands on" research and education projects that otherwise would not be practical. A commercially available affordable, high-precision, and proven turnkey automated/robotic small telescope system capable of operating remotely via the Internet is crucial for bringing this technology into widespread use. Today Torus Technologies telescopes are installed at locations worldwide as primary instruments for research programs, discovery and monitoring programs, and education programs. This paper describes some of the current applications for using these telescopes and how these telescope systems will be used in the future in standalone installations and in global networks.
\end{abstract}

\section{Introduction}

This paper describes the history of the development of robotic telescopes by Torus Technologies and what new technologies and products are planned for the near future. Before describing the development of these technologies, we need to explain our definition of what a robotic telescope actually is. 


\subsection{What are Automated and Robotic Telescopes?}

We define an automated telescope as a telescope capable of automatically following a scripted observing run pointing to objects and collecting data that are stored for later analysis. The hardware and software requirements to operate an automated telescope are modest and one or more people normally oversee the operation of the observatory. A robotic telescope extends the capabilities of an automated telescope and has the following characteristics:

- full automated control of an observatory and instruments without the need for human intervention

- system acts and reacts to feedback from environmental monitoring sensors to fulfill observing requests and protect the telescope and other equipment that are part of the observatory

- software is flexible and able to adapt to changes in the observing schedule as new events or input require new action

- remote access and control of the observatory and acquired data

A robotic telescope is a complex system that incorporates high-precision hardware, advanced electronics, and sophisticated software all of which must interoperate effectively in a system that is flexible and somewhat autonomous.

\section{The Development and Evolution of Torus Technologies Telescopes}

James Mulherin began fabricating high quality Newtonian and Cassegrain optics in the early 1990's. James and his brothers Toney Mulherin and John Mulherin designed and built their first high-precision telescopes in 1994. These first telescopes used gears and other hardware designs that were standard at the time for telescopes in the half-meter size. The Mulherin brothers used a commercially available telescope control system called PC/TCS made by COMSOFT that drove stepper motors and computer software that ran on the MS-DOS operating system. These telescopes worked well with an observer in attendance in the observatory.

\subsection{Torus Technologies' First Automațed Telescope System}

In 1995, after spending more than a year researching what was available in the industry and talking with many people knowledgeable about telescope technology, Rich Williams decided to contract James Mulherin and Torus Precision Optics (later to be renamed Torus Technologies) to design and build a 16-inch telescope for a personal observatory on his property in Western Washington. Rich planned to use his telescope and observatory to search for supernova and other monitoring projects that would require using a CCD camera for imaging and a control system that enabled automating sequences of operation. During the design phase of the project James and Rich discussed many possible configurations for the telescope design and decided on the following features: 
- Classical Cassegrain F/10 optics

- Friction rim drive system driven by stepper motors

- Equatorial fork mount

- Open-truss optical tube assembly

- A focusing mechanism that moved the secondary mirror with repeatable positioning for accurate focusing

- Components manufactured to the highest precision possible

- A control system capable of high precision tracking and pointing

Torus manufactured the telescope to the designed specifications and installed the telescope at Rich's observatory in Buckley Washington. The telescope had excellent optics and was very well made.

The original software used to control the telescope was PC/TCS, which was one of the best systems available commercially at the time. The software ran on the MS-DOS and the user interface was all text based. Rich wanted a more friendly graphical user interface with planetarium-style view to point the telescope in addition to entering object names in a form field. The owner of the software was not immediately receptive to the idea but eventually he added that capability to point the telescope using a commercially available program that ran on Microsoft Windows. However this required an additional computer to run Windows. In addition PC/TCS could not control a CCD camera and thus a third control computer was required. Although PC/TCS proved capable of running scheduled runs automatically, there were many shortcomings that soon became apparent with the overall hardware/software control system. Some of the issues that needed to be addressed included:

- Focusing and maintaining focus of the CCD camera Focusing a CCD camera on a telescope manually can be a time consuming and tedious task. PC/TCS had no capability to automatically focus a camera. Also the focus of the camera would change over time as the ambient temperature changed. During an evening scheduled run a perfect focus would gradually (sometimes rapidly) degrade until the images became poor or useless.

- Separate computers to control the telescope, CCD camera, and filter wheel Having to use separate computers with different operating systems increased the complexity and the opportunity for a failure of the system.

- Tracking and pointing of the telescope not as accurate as desired The original version of the telescope operated in an open control loop with the position being determined by counting the steps of the stepper motors. There were no encoders, hardware limit switches, or a discreet home switch. The pointing and tracking accuracy were calculated from a pointing model generated from individual stars that were centered manually during the initial setup. Overall the tracking and pointing were adequate, but not as precise as was hoped for. 


\subsection{OCAAS and the Evolution of the Automated/Robotic Telescope}

In February 1997 Rich became a partner in Torus Technologies and began working for the company full time. In early 1997 Torus decided to use the observatory control software called Observatory Control and Astronomical Analysis System (OCAAS) that the University of Iowa developed for their Automated Telescope Facility (ATF) as the telescope control system for Torus telescopes. OCAAS was a suite of software programs that controlled the ATF telescope, a CCD camera, filter wheel, and dome while checking the environment using a weather monitoring system. OCAAS operated on a single computer running the Linux operating system. In addition to its observatory control functions, OCAAS also had many image processing and data analysis capabilities and the user interface was graphical running several programs simultaneously in separate windows. The ATF was a great success and hundreds of students at the University of Iowa used the ATF as a laboratory for astronomy courses. By 1997 the University of Iowa was expanding on the AFT and proceeded with plans for the Iowa Robotic Observatory (IRO), which was a 0.5 -meter automated/robotic telescope on an alt-az mount running OCAAS for observatory control. Unlike the ATF, which was located on campus in Iowa, the IRO would be located at the Winer Mobile Observatory in Sonita Arizona and operated remotely from the University of Iowa campus. Torus worked with the development team of the IRO project fabricating the optics and consulting on some of the engineering aspects of the IRO telescope.

Rich named his observatory the Torus Observatory and the sixteen-inch Cassegrain telescope became the prototype for an evolving telescope design. OCAAS required additional hardware and electronics that the current telescopes did not have. Thus Torus retrofitted the telescope with optical encoders, hardware limit switches, and home switches for the RA, Dec, and secondary-mirror focus positions. After months of testing and debugging the telescope and CCD camera system, OCAAS proved to be a very powerful and effective control system. The Torus Observatory used an Apogee AP7 CCD camera with a 512 x 512 SITe back-illuminated CCD chip with 24-micron pixels, which provided a 10.5 arc-minute by 10.5 arc-minute field of view. Although the weather in Western Washington is notoriously cloudy, Rich managed to get impressive results from the system and began doing asteroid astrometry work for the Minor Planet Center, working on other projects, and demonstrating the telescope to prospective customers from around.

\subsection{Birth of the Classical Cassegrain Series Telescopes}

The Torus Observatory telescope proved to be so successful that Torus decided to use OCAAS and the features incorporated in the telescope as the basis for the development of all the company's telescopes and the standard was set for future research and development. Torus decided to market a line of telescopes based on OCAAS and the prototype system built for the Torus Observatory. This line of telescopes was called the TORUS Classical Cassegrain Series and the telescopes have common features: OCAAS, F/10 Classical Cassegrain optical systems, equatorial fork mounts fabricated from aluminum, friction-rim drives, and so on. Standardizing on major parts of the telescope systems was 
an important business decision for Torus. Rather than producing each telescope as a "one off" development project with a large investment in engineering time for research and development, the main components would be designed and the production blueprints would be used over and over again. This approach cut the development and production time for manufacturing telescopes considerably and reduced the cost making the company more profitable for investors.

Beginning in 1997 members of the Torus staff started attending and exhibiting at major astronomy meetings and workshops around the world. With the success of the Torus Observatory telescope and OCAAS, the astronomy community started to take notice of what Torus was doing. Torus sold the first TORUS Classical Cassegrain Series telescope in early 1998, and then other innovative customers that believed in our ideas and telescope designs, showed interest and ordered telescopes. To handle the increased production, Torus moved to a larger facility. Eventually Torus standardized on three different mount designs for the telescopes in the Classical Cassegrain Series ranging from 0.4-meters to 1.0-meters in diameter. The general specifications of the hardware and software are common to all telescopes in the series.

\subsection{Development of the Wide-Field Hybrid Cassegrain Telescope System}

Rich Williams and John Mulherin attended the UN/ESA Workshop on Basic Space Science in Tegucigalpa Honduras in March 1997. At the workshop they met Dr. Syuzo Isobe from Japan who talked with them about his plans for a project that would require a 0.5 -meter and a 1.0-meter wide field telescope to search for and monitor near-earth objects (NEOs). Ultimately Torus won the bid for the telescopes and designed a new type of catadioptric Cassegrain telescope. The optical configurations of the telescopes were a Cassegrain design with several refractive elements (lenses) to achieve focal ratios of $F / 1.9$ for the 0.5 -meter telescope and F/3 for the 1.0-meter telescope. The telescopes were designed to achieve a very wide filed of view to cover as much of the sky as possible in the shortest amount of time using new CCD cameras with a mosaic of CCD chips with $2 \mathrm{k} \times 4 \mathrm{k}$ pixels. The 0.5 -meter telescope was designed for a 2-degree field of view and the 1.0-meter telescope was designed for a 3-degree field of view. Because we needed a name for this new design, we decided to call them wide-field hybrid Cassegrain telescopes. Shortly after starting Dr. Isobe's project for the Bisei Spaceguard Center telescopes, Torus discovered that there was a great deal of interest in the wide-field hybrid Cassegrain telescope design. These new telescopes were ideal for doing survey and monitoring projects automatically. In 1999 Torus won contracts to build several more 0.5 -meter widefield hybrid Cassegrain telescopes for the Taiwan-America Occultation Survey (TAOS) project and the Yonsei Survey Telescopes for Astronomical Research (YSTAR) project. Because we had several orders for these telescopes virtually at the same time, we treated them as a "production run" using as much of the engineering as possible from the Classical Cassegrain Series designs. Because the optical tube assembly part of these telescopes required new research and development, producing several of these telescopes at more or less the same time caused problems that we did not foresee. The optical design of the wide-field hybrid Cassegrain telescopes is very challenging to fabricate, align, and test 
requiring the development of new assembly and test equipment. The actual implementation of the design proved to be much more difficult than we anticipated. However, we eventually resolved the problems. One of the greatest challenges with this optical design is collimating the optics. Because the optical system is very fast and incorporates refractive elements, we had to devise new collimation procedures for these systems.

\subsection{Project Rigel}

Dr. Robert Mutel is the principal astronomer responsible for the idea and implementation of the very successful ATF and IRO projects at the University of Iowa. Dr. Mutel is one of the leading experts on using robotic telescope systems for education and research. Hundreds of students have used the ATF and now the IRO for astronomy laboratory work. Based on the success of these projects, Dr. Mutel applied for and received an NSF grant to contract with Torus Technologies to produce an affordable turnkey robotic observatory system based on the work and lessons learned from the ATF and the IRO projects. The purpose of the project is to produce the prototype for a commercial product that Torus can sell and make widely available to institutions and people around the world. Project Rigel is a robotic astronomy laboratory. The following is a description of the Rigel Project published on the University of Iowa's web site:

"The Rigel Project is a robotic observatory designed for use in undergraduate astronomy education and research. It is a complete turnkey system consisting of a 14.5-inch f/14 Cassegrain telescope, large-format CCD camera, filter wheel, spectrometer, dome, and weather station. The observatory control software is Web-based and highly user-friendly. The observatory can be operated in 'real-time' or by queue-based scheduling. The prototype Rigel system is under construction now, and will be tested during the summer and fall of 2001. Torus expects to begin delivery of the first production Rigel systems in the spring of 2002.

A companion curriculum is being developed which will allow instructors to integrate Rigel observations into astronomical laboratories. Although the primary focus will be undergraduate laboratories, parts of the curriculum will be suitable for high school courses, as well as more advanced undergraduate research projects."

As part of the project Rigel development, OCAAS will be enhanced and made more powerful and new web based software tools will make operating and using Torus robotic telescopes even easier.

\subsection{A Worldwide Network of Robotic Telescopes and Other Future Developments}

Although the Rigel project is in development and will not be an actual product until early 2002, Torus is looking beyond Rigel to the next phase of the evolution of our telescope technology. We plan to expand on the web-based and OCAAS software from the Rigel project to develop a fully integrated worldwide telescope network based on OCAAS and the web browser tools developed for the Rigel project. Starting with the new Torus Observatory in California and our current 
customers, we will test and build a flexible and robust global telescope network. We will not limit this network solely to Torus Technologies telescopes. Part of our plan to make the global network grow and thrive is to make OCAAS readily available to retrofit existing telescopes and make them compatible to operate among the telescopes in the global network. Ultimately at the highest level of the worldwide network we want to enable any telescope to "plug into" the network independent of what telescope control system it uses. This will most likely be accomplished using a platform independent protocol such as the proposed Robotic Telescope Markup Language (RTML), which is a superset of XML. As new technologies and opportunities arise, Torus Technologies will develop new technologies and products and make them commercially available to the astronomy community.

\section{References}

Torus Technologies: www.torusoptics.com

University of Iowa AFT and IRO projects: denali.physics.uiowa.edu

Bisei Spaceguard Center: www.spaceguard.or.jp/bsgc/pamphlet/index.htm

TAOS project: taos.asiaa.sinica.edu.tw

Project Rigel: denali.physics.uiowa.edu/rigel/ 

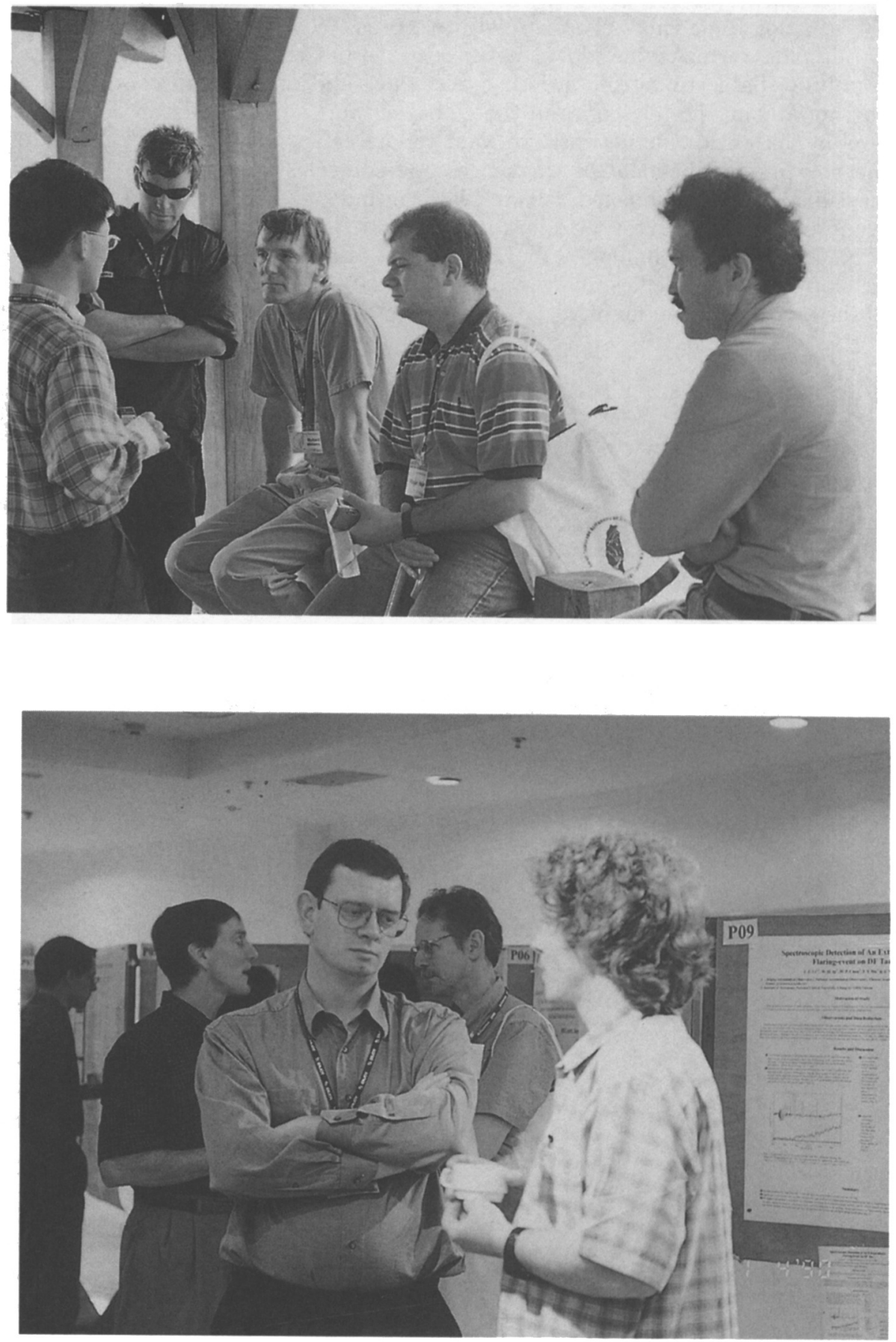

(Top from left) You, Rattenbury, Williams, Hajjar, Hojaev; (bottom from left) Mack, Gelderman (front), Williams, Alcock (back) 